\title{
Microchemical Engineering in 3D Ordered Channel Enhances Electrocatalysis
}

Qing-Xia Chen, ${ }^{\mathrm{a}, \dagger}$ Ying-Huan Liu, ${ }^{\mathrm{b}, \dagger}$ Zhen He, ${ }^{\mathrm{a}}$ Jin-Long Wang, ${ }^{\mathrm{a}}$ Jian-Wei Liu, ${ }^{\mathrm{a}, *}$ Hui-Jun Jiang, b,* Wei-Ran Huang, ${ }^{a}$ Guan-Yin Gao, ${ }^{a}$ Zhong-Huai Hou, ${ }^{b}$ and ShuHong $\mathrm{Yu},{ }^{\mathrm{a}, *}$

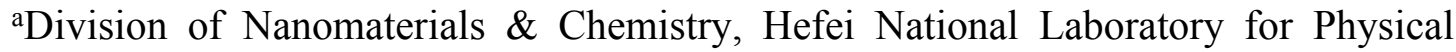
Sciences at the Microscale, Institute of Energy, Hefei Comprehensive National Science Center, CAS Center for Excellence in Nanoscience, Department of Chemistry, Institute of Biomimetic Materials \& Chemistry, Anhui Engineering Laboratory of Biomimetic Materials, University of Science and Technology of China, Hefei 230026, China. bDepartment of Chemical Physics \& Hefei National Laboratory for Physical Sciences at Microscales, iChEM, University of Science and Technology of China, Hefei 230026, China

* Correspondence and requests for materials should be addressed to the author: JianWei Liu (jwliu13@ustc.edu.cn), Hui-Jun Jiang (hjjiang3@mail.ustc.edu.cn) or ShuHong Yu (shyu@ustc.edu.cn). 


\section{Contents}

\section{Supplementary Figures}

Figure S1. Mass transfer of $\mathrm{MeOH}$ molecular from the bulk electrolyte to the near surface area of electrode.

Figure S2. The numerical simulation setup.

Figure S3. Morphological characterizations of Te NWs and Pt NTs.

Figure S4. Schematic diagram of the preparation of 3DOC by Langmuir-Blodgett assembly technique and in-situ electrochemical etching.

Figure S5. Compositional determination and structural characterizations of the 3DOC. Figure S6. Morphological characterizations of co-assembled monolayers and crosslinked bilayers.

Figure S7. Methanol oxidation reaction (MOR) measurements and morphological characterizations of 3DOC.

Figure S8. Morphological characterizations of 3DOC.

Figure S9. Schematic diagram of 3DOC model on the surface of GCE and electrochemical double layer (EDL) with various channel sizes.

Figure S10. The methanol transfer flux on electrode modified with disordered NTs.

Figure S11. Morphological characterizations of large-scaled monolayer from various $\mathrm{Pt}$ NTs/Te NWs ratios.

Figure S12. Quantitative and spectral characterizations of 3DOC with various channel sizes.

Figure S13. Schematic diagrams and the small angle X-ray scattering (SAXS) patterns of Pt NT monolayers and crossed bilayers various separation distances.

Figure S14. Reaction rates in the conditions of stable electron transfer ability and enhanced electron transfer ability with increased channel sizes.

Figure S15. The water contact angles of 3DOC with various channel sizes.

Figure S16. Schematic diagram of the contact angle as the channel sizes change.

Figure S17. MOR performances of 3DOC and corresponding disordered accumulations. Figure S18. Stability characterization of 3DOC.

Figure S19. The generality of MCE strategy.

\section{Supplementary Tables}

Table S1. Selection of the parameters in MOR at the 3DOC of $40 \mathrm{~nm}$.

\section{Supplementary Movies}

Movie S1. The concentration distribution evolution of $\mathrm{MeOH}$ on 3DOC-1.

Movie S2. The concentration distribution evolution of $\mathrm{MeOH}$ on 3DOC-2.

Movie S3. The concentration distribution evolution of $\mathrm{MeOH}$ on 3DOC-3.

Movie S4. The concentration distribution evolution of $\mathrm{MeOH}$ on 3DOC-4.

Movie S5. The concentration distribution evolution of $\mathrm{MeOH}$ on disordered counterpart. 


\section{Supplementary Figures}

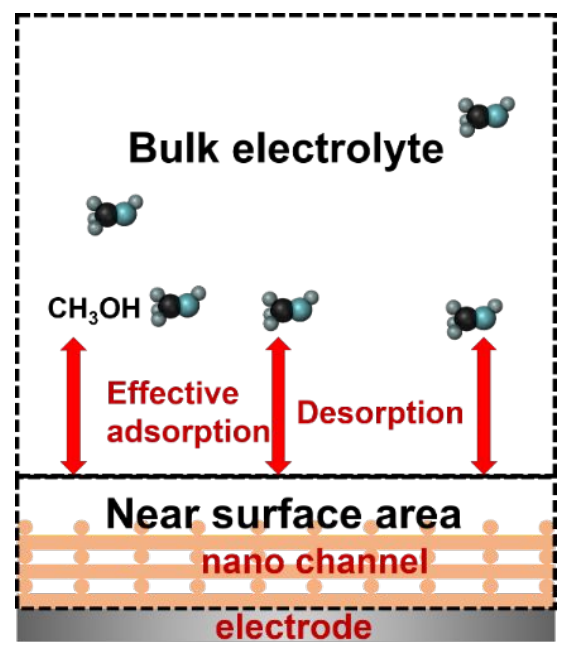

Figure S1. Mass transfer of $\mathrm{MeOH}$ molecular from the bulk electrolyte to the near surface area of electrode. Based on the free energy density function of the adsorbed $\mathrm{MeOH}$ molecular near the electrode surface, a comprehensive kinetic model coupling the mass transfer and surface reaction was established. During the MOR process, $\mathrm{MeOH}$ firstly diffuses from the bulk electrolyte to near the electrode, and then adsorbs onto the electrode, eventually is oxidized on the electrode.

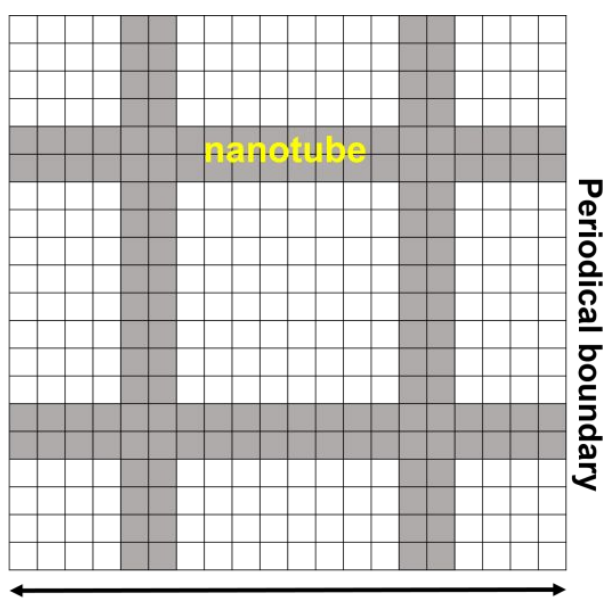

L

Figure S2. The numerical simulation setup. Schematic of electrode surface discretized in 2D $L \times L$ lattice with periodical boundary conditions. The gray pixels indicate where the nanotubes are located. 

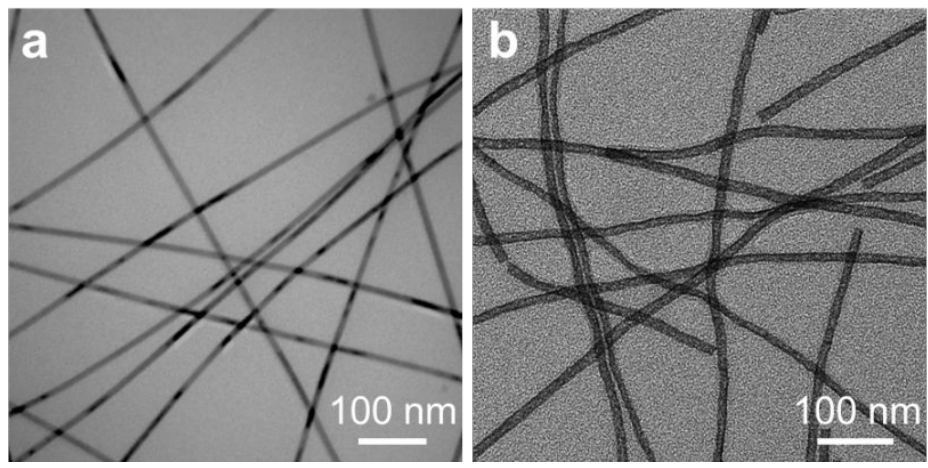

Figure S3. Morphological characterizations of Te NWs and Pt NTs. Representative TEM images of (a) sacrificial template Te NWs, and (b) Pt NTs. As the figure S3a shows, Te NWs are ultrathin and highly uniform with a diameter of about $7 \mathrm{~nm}$. And Pt NTs have an inner diameter of $4 \mathrm{~nm}$ and a wall thickness of $2 \mathrm{~nm}$, respectively, shown in figure $\mathrm{S} 3 \mathrm{~b}$.
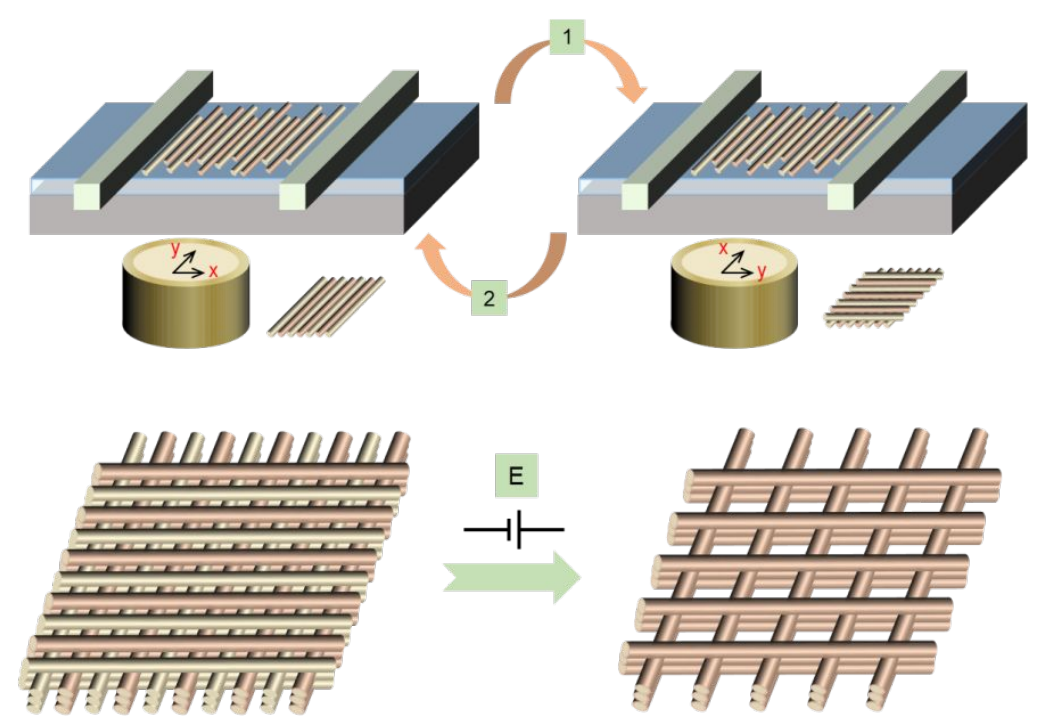

Figure S4. Schematic diagram of the preparation of 3DOC by Langmuir-Blodgett assembly technique and in-siut electrochemical etching. The yellow and orange sticks indicate Te NWs and Pt NTs, respectively. The cylinder represents a detachable glassy carbon electrode (GEC). After a compact monolayer co-assembly is deposited onto the GEC and dried, the GEC is rotated $90^{\circ}$ before transferring the second layer. With this process repeated three times, a 3D ordered network of Te NWs and Pt NTs is constructed. Te NWs are electrochemically oxidated during the current voltage (CV) cycles in acidic medium at the voltage window from -0.2 to $1.05 \mathrm{~V}$. With Te NWs electrochemically etched away, 3DOC is eventually formed. 

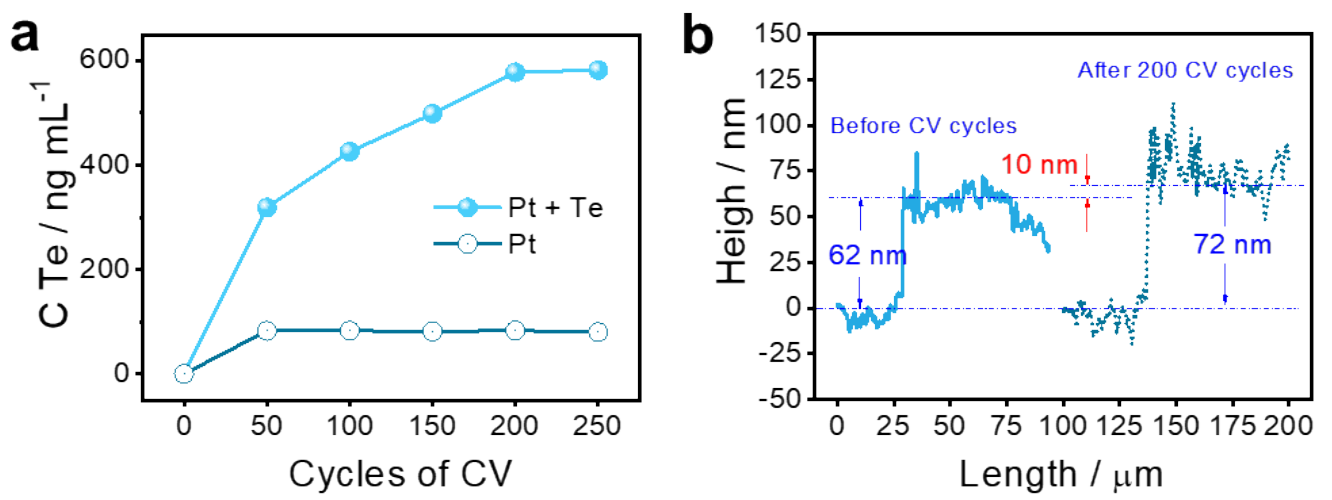

Figure S5. Compositional determination and structural characterizations of the 3DOC. (a) Tellurium mass in the electrolyte during CV cycles in Pt NT and Pt NT/Te NW coassembles measured from ICP-MS. CV cycles are conducted in $0.1 \mathrm{M} \mathrm{HClO}_{4}$ at a scan rate of $250 \mathrm{mV} \mathrm{s}^{-1}$. The initial increase of Te content in Pt NT network is due to the residual Te in Pt NTs that is not been completely replaced in Kirkendall reaction between Te NWs and Pt NTs. In order to make the ICP-MS test results more accurate, the same volume of electrolyte is added after every sampling. (b) Height measurement of 3DOC-2 before and after $200 \mathrm{CV}$ cycles from a step profiler. The results show that the surface of 3DOC is relatively flat. After Te NWs are electrochemically etched, the network structure of 3DOC remains robust, which provides the basis for the subsequent MOR measurement.
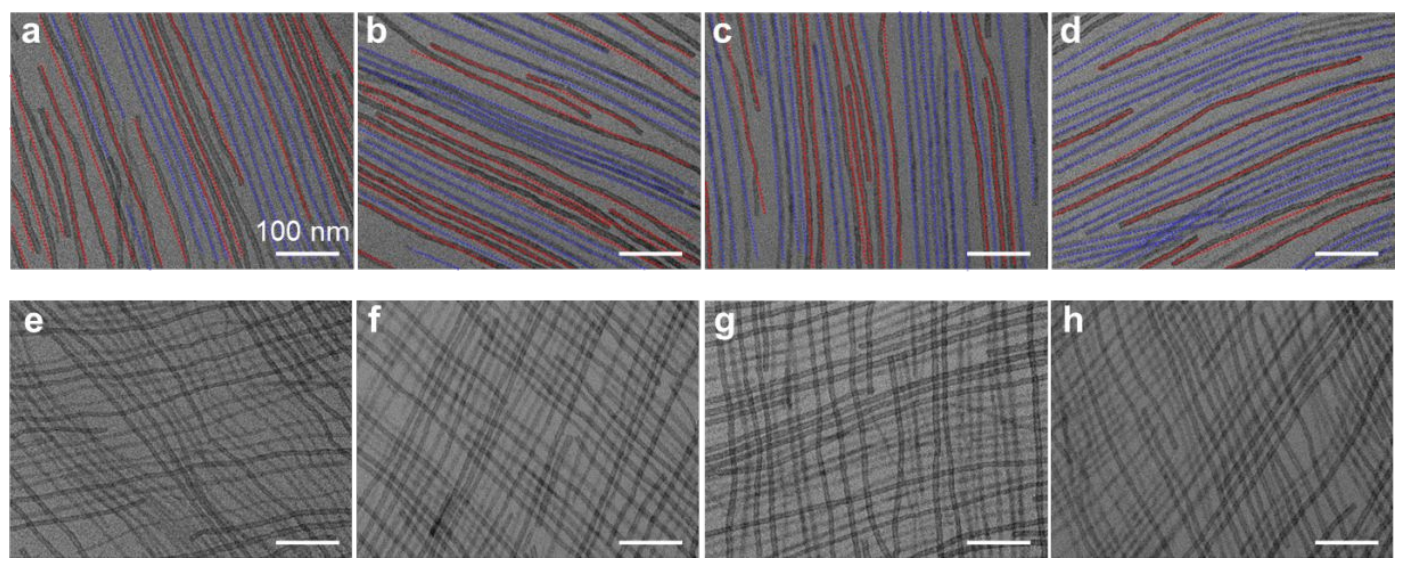

Figure S6. Morphological characterizations of co-assembled monolayers and crosslinked bilayers. Representative TEM images of (a-d) co-assembled monolayers and (eh) cross-linked bilayers. The Pt NTs/Te NWs ratios in (a-d) are 2/1/0.67/0.5, respectively. Red and blue dashes indicate Pt NTs and Te NWs, respectively. All the scale bars are $100 \mathrm{~nm}$. 

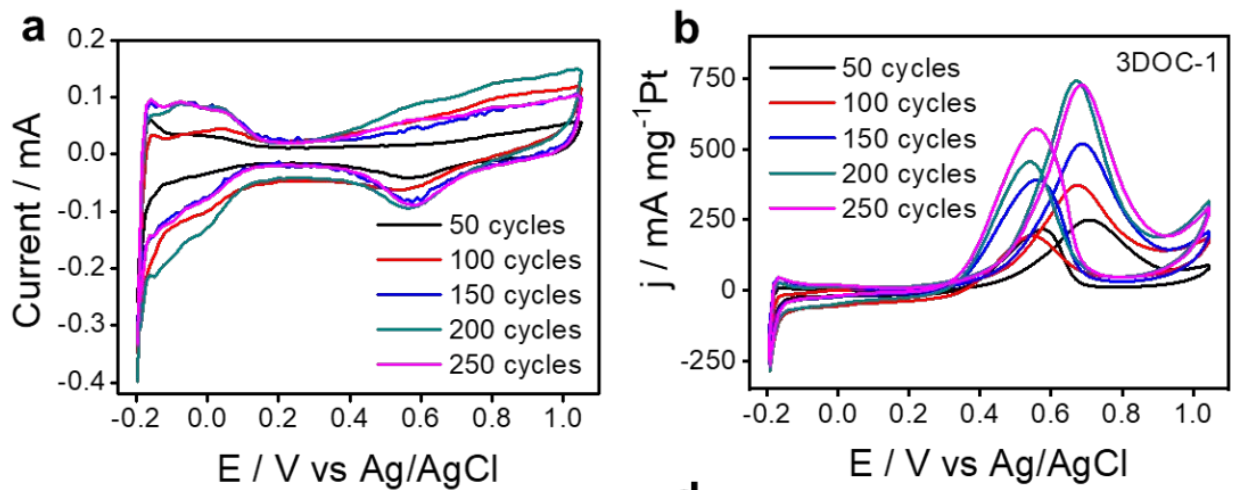

C
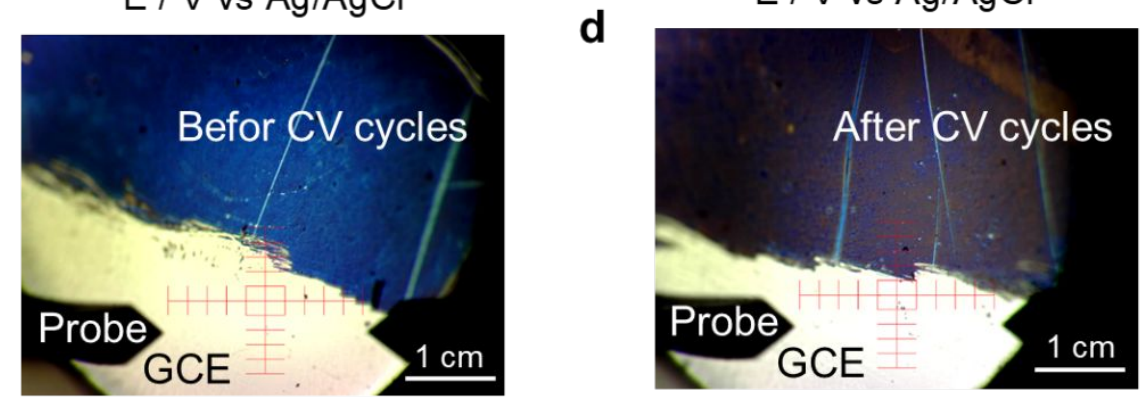

Figure S7. Methanol oxidation reaction (MOR) measurements and morphological characterizations of 3DOC. (a) CV curves of 3DOC-2 with various cycles in $0.1 \mathrm{M}$ $\mathrm{HClO}_{4}$ at a scan rate of $50 \mathrm{mV} \mathrm{s}^{-1}$. With the increase of $\mathrm{CV}$ cycles, the hydrogen evolution peak becomes more and more obvious and reaches the maximum at 200 cycles. At 250 cycles, the peak area of hydrogen evolution is even reduced. Therefore, the electrochemical etching and activation is selected at 200 cycles. (b) MOR curves of $3 \mathrm{DOC}-2$ with various cycles in $0.1 \mathrm{M} \mathrm{HClO}_{4}+1.0 \mathrm{M} \mathrm{CH}_{3} \mathrm{OH}$ at a scan rate of $50 \mathrm{mV}$ $\mathrm{s}^{-1}$. The relationship between mass activities and cycle numbers also shows a similar trend as the hydrogen evolution peak areas. (c, d) Optical photographs of 3DOC-2 on the detached glassy carbon electrode (GCE) before and after $200 \mathrm{CV}$ cycles. The scratches are caused by the probe of the step profiler.
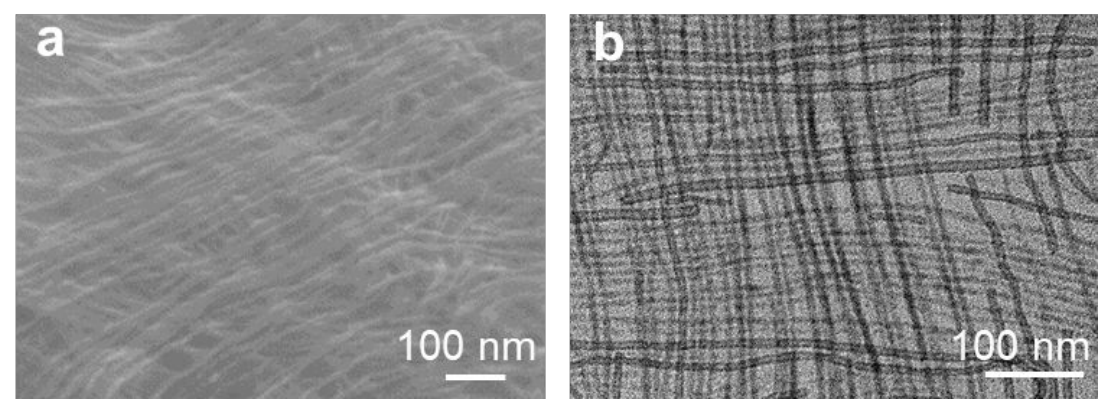

Figure S8. Morphological characterizations of 3DOC. Representative (a) SEM, and (b) TEM images of 3DOC with six orthogonal Pt NT layers. The results further confirm that 3DOC has a flat surface, as well as an opened channel structure. 
a

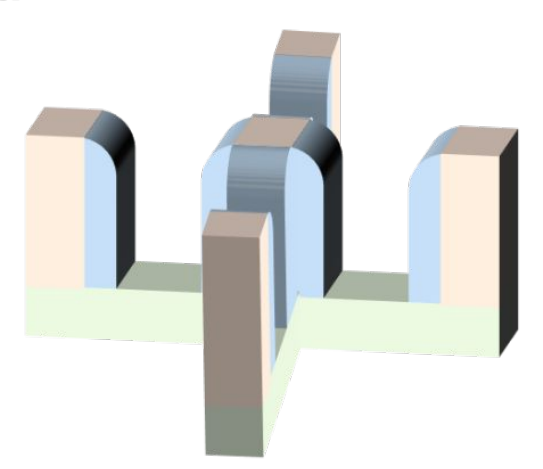

b NT EDL

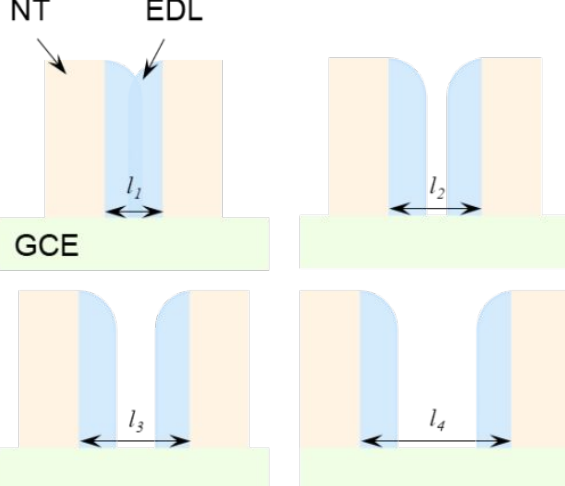

Figure S9. Schematic diagram of 3DOC model on the surface of GCE and electrochemical double layer (EDL) with various channel sizes. (a) Schematic diagram of 3DOC model on the surface of GCE. GCE, NT, and EDL are indicated in green, orange, and blue, respectively. Channels in 3DOC are ordered and connected to each other. (b) EDL of $3 \mathrm{DOC}$ with various channel sizes. $l_{n}$ is the channel size. The thickness of EDL is determined by the properties of catalyst and electrolyte, such as ionic strength and permittivity. EDL overlaps in small aperture while separates in large one, which greatly influences the diffusion of molecules into the surface of catalyst and then the kinetics of the whole reaction throughout the channel.

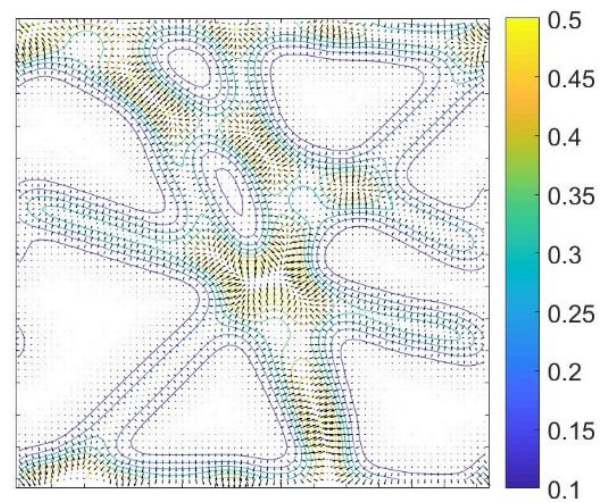

Figure S10. The methanol transfer flux on electrode modified with disordered NTs. Black arrows show the direction of $\mathrm{MeOH}$ flux while the contour line is the distribution of flux velocity. 


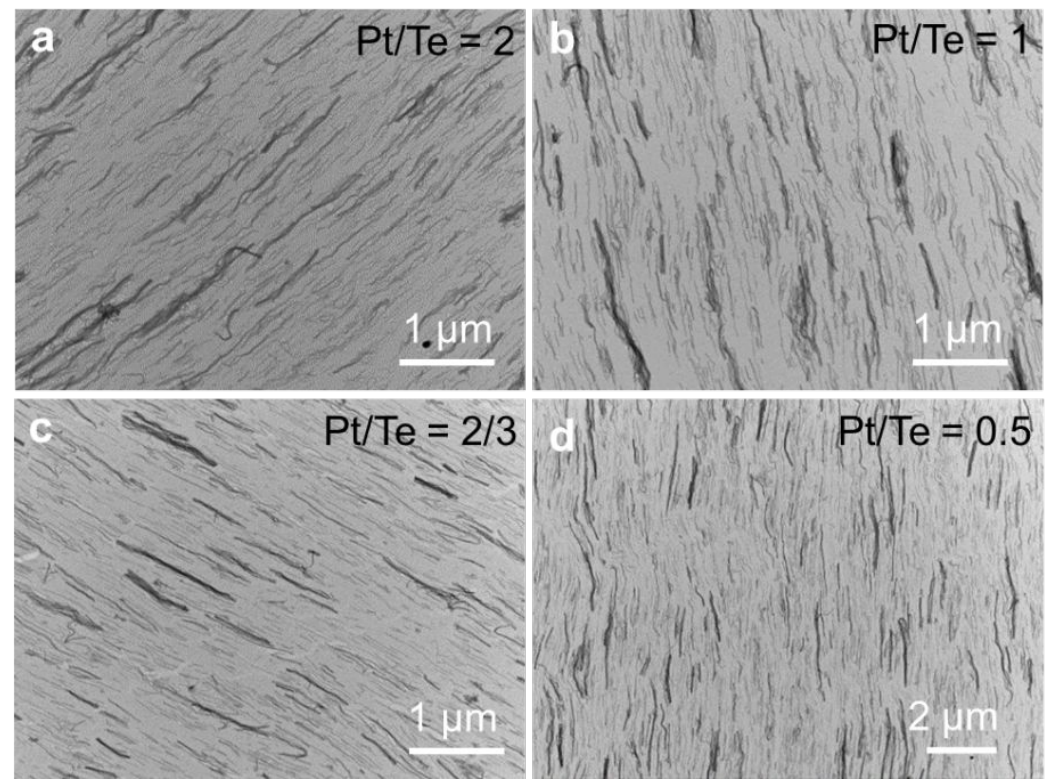

Figure S11. Morphological characterizations of large-scaled monolayer from various Pt NTs/Te NWs ratios. This shows that the co-assembled monolayer is continuous and the surface is relatively even and smooth.
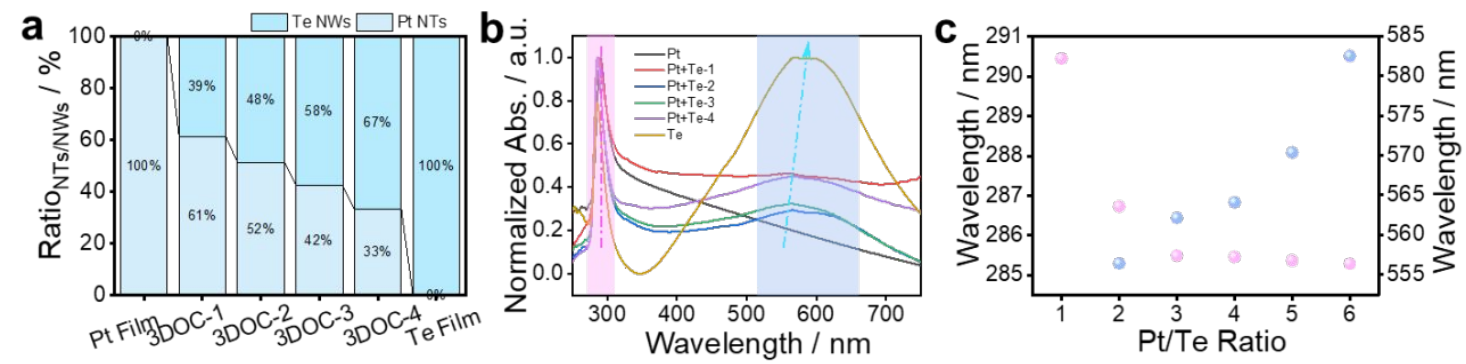

Figure S12. Quantitative and spectral characterizations of 3DOC with various channel sizes. (a) Statistical results of Pt NTs/Te NWs ratios from representative TEM images of co-assembled monolayers with various $\mathrm{Pt}$ NTs/Te NWs ratios in assembly ink. $\mathrm{Pt}+\mathrm{Te}-1 / 2 / 3 / 4$ refer to the $\mathrm{Pt} \mathrm{NTs} / \mathrm{Te} \mathrm{NWs}$ ratios in assembly ink of $2 / 1 / 0.67 / 0.5$. (b) UV-vis spectra of the co-assembled monolayers with various Pt NTs/Te NWs ratios in comparision with Pt NTs and Te NWs. (c) The peak locations varying with the Pt NTs/Te NWs ratios near 285 and $570 \mathrm{~nm}$. These two peaks correspond with the characteristic peak of Pt NTs and Te NWs, respectively. 


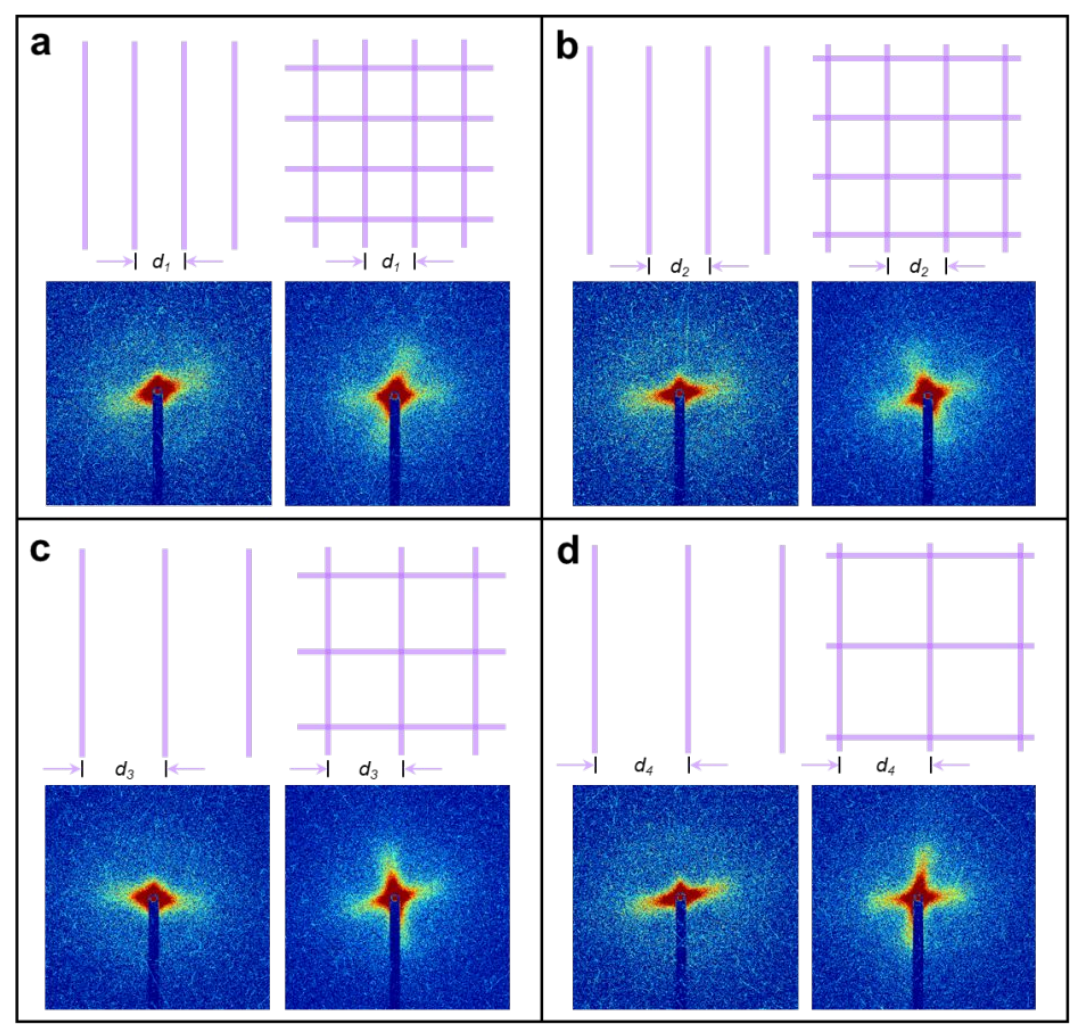

Figure S13. Schematic diagrams and the small angle X-ray scattering (SAXS) patterns of Pt NT monolayers and crossed bilayers various separation distances. (a) $d_{1}=40 \mathrm{~nm}$; (b) $d_{2}=60 \mathrm{~nm}$; (c) $d_{3}=90 \mathrm{~nm}$; (d) $d_{4}=120 \mathrm{~nm}$. These results indicate that Pt NTs are aligned orderly in a unidirectional direction in each monolayer of 3DOC. And the adjacent layers intersect at $90^{\circ}$ shown by the orthogonal scattering directions.
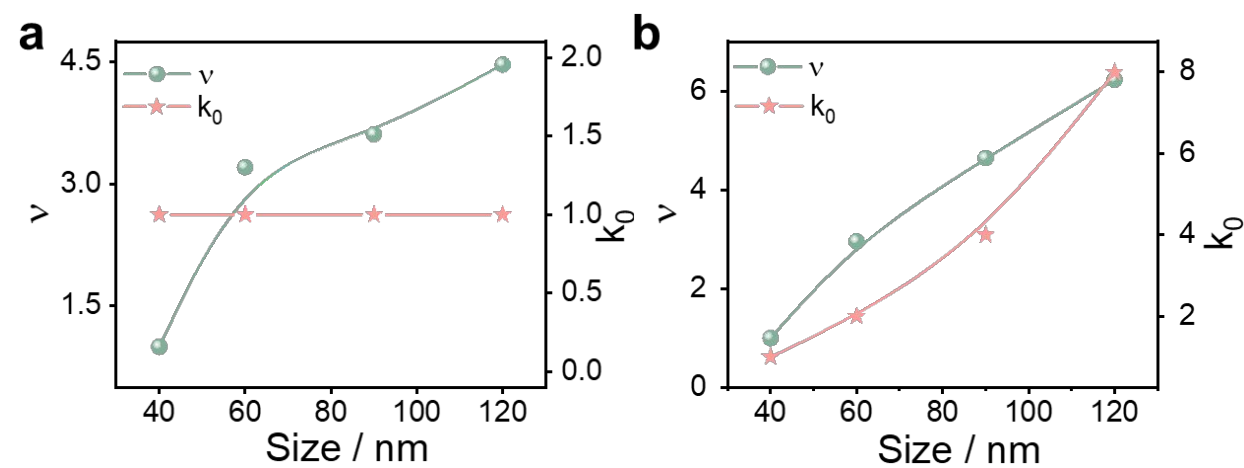

Figure S14. Reaction rates in the conditions of stable electron transfer ability and enhanced electron transfer ability with increased channel sizes. $v$ is the reaction rate while $k_{0}$ is the reaction rate constant. As we suppose that the electron transfer ability is decreased with an increasing $l$, other conditions such as increased electron transfer ability (b) or unchanged electron transfer ability (a) with the increased $l$ are also considered. The results indicate that the total reaction rate is continually increasing with the enlarged channel, which is the result of corporation between increased mass transfer and increased/unchanged electron transfer ability. 


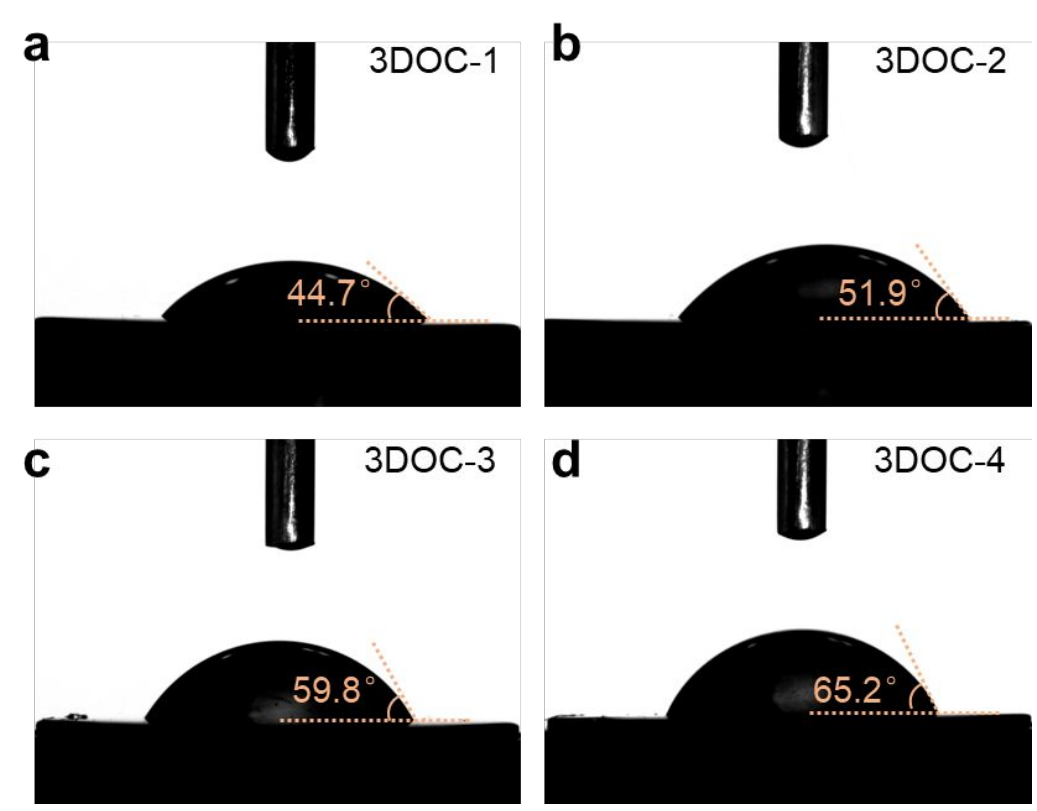

Figure S15. The water contact angles of $3 \mathrm{DOC}$ with various channel sizes. With the increase of channel size, the water contact angles of the 3DOC increases.

a

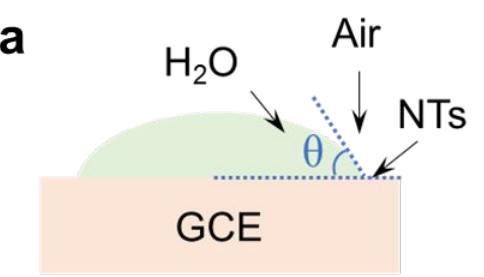

glassy carbon electrode

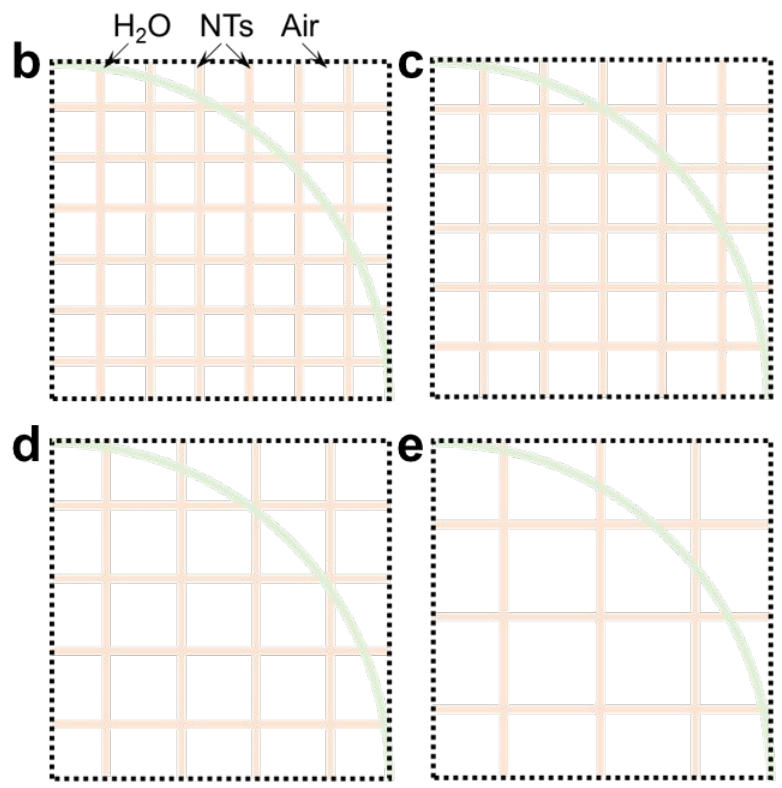

Figure S16. Schematic diagram of the contact angle as the channel sizes change. (a) Schematic diagrams of the three-phase contact line in 3DOC. (b-e) The top views of the three-phase contact line in 3DOC with various channel sizes. As the channel size increases, the air trapped inside the channel becomes more concentrated, which makes the water contact angle larger. 

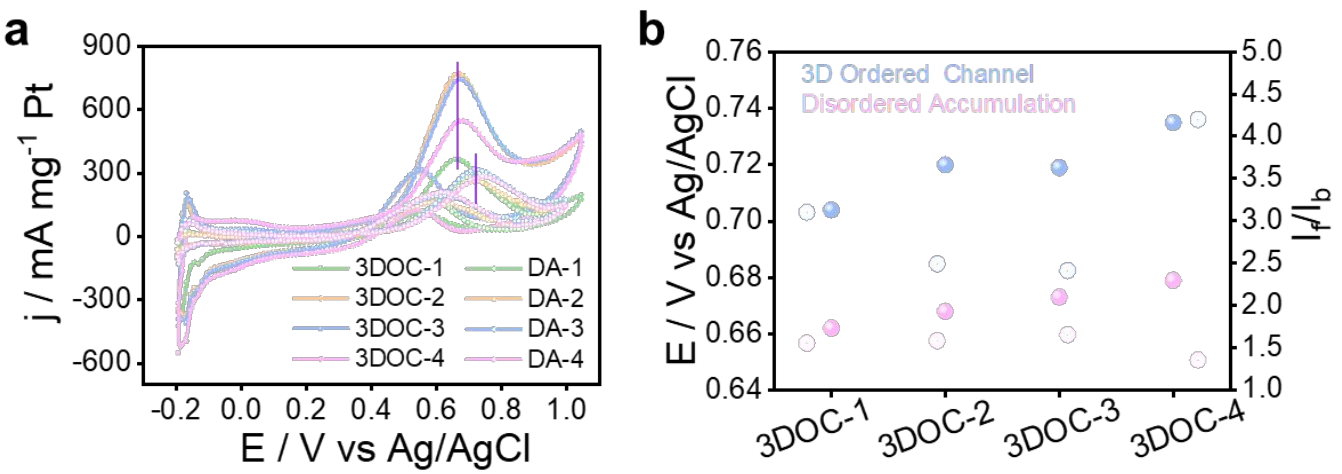

Figure S17. MOR performances of $3 \mathrm{DOC}$ and corresponding disordered accumulations. (a) MOR profiles of 3DOC with various channel sizes in comparision with disordered accumulations. The measurements were carried out in $0.1 \mathrm{M} \mathrm{HClO}_{4}+$ $1.0 \mathrm{M} \mathrm{CH}_{3} \mathrm{OH}$ after 50 cycles of $\mathrm{CV}$ activation at a scan rate of $50 \mathrm{mV} \mathrm{s}^{-1}$. (b) $\mathrm{E}_{\text {peak }}$ forward and If $/ \mathrm{I}_{\mathrm{b}}$ comparisons of $3 \mathrm{DOC}$ with various channel sizes in comparision with disordered accumulations.

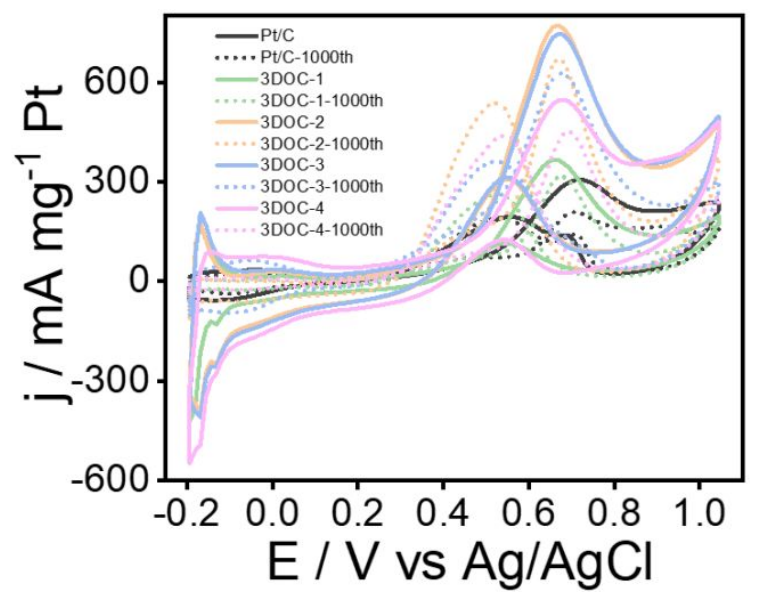

Figure S18. Stability characterization of 3DOC. MOR profiles of the first and $1000^{\text {th }}$ cycles in comparasion with $\mathrm{Pt} / \mathrm{C}$. 

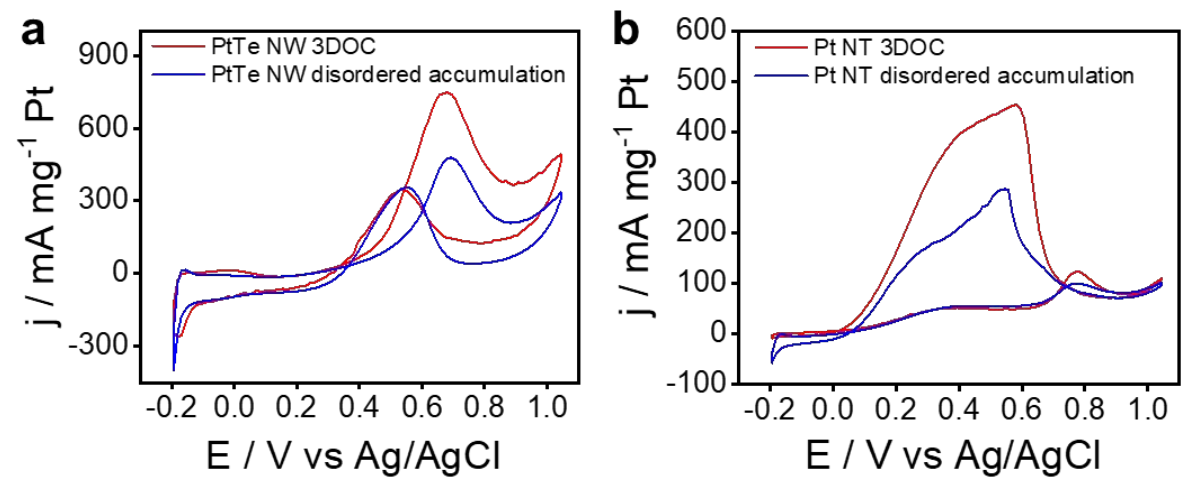

Figure S19. The generality of MCE strategy. a, MOR performances of PtTe NW 3DOC and their disordered accumulation in $0.1 \mathrm{M} \mathrm{HClO}_{4}+1.0 \mathrm{M} \mathrm{CH}_{3} \mathrm{OH}$ at a sweep rate of $50 \mathrm{mV} \mathrm{s}^{-1} . \mathrm{b}$, Formic acid oxidation reaction (FAOR) performances of Pt NT 3DOC and their disordered accumulation in $0.1 \mathrm{M} \mathrm{HClO}_{4}+1.0 \mathrm{M} \mathrm{HCOOH}$ at a sweep rate of $50 \mathrm{mV} \mathrm{s}^{-1}$. Error bars correspond to the standard deviations taken over at least three measurements. 


\section{Supplementary Tables}

Table S1. Selection of the parameters in MOR at the 3DOC of $40 \mathrm{~nm}$.

\begin{tabular}{cccccccccc}
\hline Parameters & $\mathrm{D}$ & $\mathrm{N}$ & $\boldsymbol{k}_{\boldsymbol{a d}}$ & $\boldsymbol{k}_{\boldsymbol{d e}}$ & $\alpha$ & $\lambda$ & $\boldsymbol{k}_{\boldsymbol{\eta}=\mathbf{0}}$ & $\boldsymbol{u}_{\mathbf{0}}$ & $\eta$ \\
\hline Values & 1.0 & 72 & 1.0 & 9.0 & 0.12 & 2.0 & 8.0 & 1.0 & 1.1
\end{tabular}

Supplementary Movies 1-5. 